\title{
BMJ Open Multimorbidity in primary care: protocol of a national cross-sectional study in Switzerland
}

Anouk Déruaz-Luyet, ${ }^{1}$ A Alexandra N'Goran, ${ }^{1}$ Ryan Tandjung, ${ }^{2}$ Peter Frey, ${ }^{3}$
Andreas Zeller, ${ }^{4}$ Dagmar M Haller, ${ }^{5}$ Thomas Rosemann, ${ }^{2}$ Bernard Burnand,
Patrick Bodenmann, ${ }^{7}$ Nicolas Senn, ${ }^{7}$ Daniel Widmer, ${ }^{1}$ Lilli Herzig ${ }^{1}$

To cite: Déruaz-Luyet $A$, N'Goran AA, Tandjung R, et al. Multimorbidity in primary care: protocol of a national cross-sectional study in Switzerland. BMJ Open 2015;5:e009165.

doi:10.1136/bmjopen-2015009165

- Prepublication history for this paper is available online To view these files please visit the journal online (http://dx.doi.org/10.1136/ bmjopen-2015-009165)

Received 22 June 2015 Revised 4 September 2015 Accepted 14 September 2015

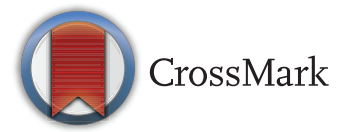

For numbered affiliations see end of article.

Correspondence to Dr Anouk Déruaz-Luyet; anouk.deruaz-luyet@hospvd. ch

\section{ABSTRACT}

Introduction: With the ageing of the population and the general improvement of care, an increasing number of people are living with multiple chronic health conditions or 'multimorbidity'. Multimorbidity often implies multiple medical treatments. As a consequence, the risk of adverse events and the time spent by patients for their treatments increase exponentially. In many cases, treatment guidelines traditionally defined for single conditions are not easily applicable. Primary care for individuals with multimorbidity requires complex patient-centred care and good communication between the patient and the general practitioner (GP). This often includes prioritising among the different chronic conditions.

Methods and analysis: The main objectives of this study are to describe the burden related to multimorbidity (disease-related burden and burden of treatment) in primary care and to identify the factors influencing it. Other objectives include evaluating patients' perception of treatment burden and quality of life, assessing factors influencing that perception, and investigating prioritisation in the management of multimorbidity from the perspectives of GPs and patients. For this crosssectional study, patient enrolment will take place in GP's private practices across Switzerland. A convenient sample of $100 \mathrm{GPs}$ will participate; overall, 1000 patients with at least three chronic health conditions will be enrolled. Data will be collected as paper-based questionnaires for patients. GPs will provide demographic and practice-related data. In addition, each GP will complete a paper-based questionnaire for each patient that they enrol. Each patient will complete a telephone interview questionnaire.

Ethics and dissemination: This study has been approved by the research ethics committee of Canton Vaud, Switzerland (Protocol 315/14). The results of the study will be reported in international peer-reviewed journals.

\section{INTRODUCTION}

Prevalence of multimorbidity in primary care Multimorbidity is defined as the co-occurrence of chronic medical conditions within questionnaires for GPs and delayed telephone interview one person without a single condition being considered the main condition. ${ }^{1}$ Caring for multimorbid patients is an important part of general practice. A large population-based study conducted in the UK estimated that $42.2 \%$ of the population visiting a general practitioner (GP), including children, had at least one chronic health condition; $23.2 \%$ had at least two chronic conditions. ${ }^{2}$ Other studies estimated the prevalence of multimorbidity in primary care between $13 \%$ and $75.5 \%$ depending on the population, the age group considered, and the definition of multimorbidity applied. ${ }^{3-8}$ The prevalence of multimorbidity is influenced by different factors, including age and socioeconomic status (SES). ${ }^{2} 9{ }^{10}$ Multiple chronic conditions are more prevalent in the elderly, and multimorbidity develops $10-15$ years earlier in the most deprived areas. ${ }^{211}$ The ageing of the population, together with the improvement of medical services, has led to the increased survival of people with chronic conditions and to an increased prevalence of multimorbidity. ${ }^{12}$ Uijen and Van de Lisdonk ${ }^{12}$ observed, on data from a registry including 10 GPs and approximately 13500 patients, that the proportion of patients with at least four chronic conditions tripled between 1985 and 2005 .

\section{Disease-related burden and burden of treatment}

Cumulative effects of multiple chronic conditions can be expressed in terms of disease-related burden, or burden of treatment. Studies suggest that the cumulative effect of chronic health conditions is not simply incremental; specific combinations of conditions may have a greater effect on functional status, quality of life and mortality than others. ${ }^{13} 14$ The burden of treatment itself encompasses different aspects, such as 
the number of investigations, number of pills to take each day, number of injections to take, number of check-ups or preventive measures, and other care (eg, education or physiotherapy). For instance, an estimated $20 \%$ of older adults in the US population take more than 10 different medications each week. ${ }^{15}{ }^{16}$ Owing to the importance of the disease-related burden and burden of treatment, primary care for people with multimorbidity must address disease-specific issues in the context of coexisting chronic medical conditions and the patient's psychosocial environment. This implies complex, patient-centered care involving patient-provider concordance, effective communication and shared decision-making. ${ }^{17} 18$ One of the main difficulties GPs face is the challenge of following guidelines that are typically developed for single diseases. ${ }^{19-21}$ Sinnott $e t a t^{2}$ synthesised qualitative research on the perception that GPs and other primary care practitioners have of the management of multimorbidity. Ten studies conducted in seven countries (Belgium, England, Germany, Ireland, Scotland, the Netherlands and the USA), including 275 GPs, were considered. The synthesis emphasised that difficulties in the care of people with multimorbidity mainly arise from disorganisation and fragmentation of healthcare, inadequacy of guidelines and evidence-based medicine, and challenges to patientcentered care and shared decision-making. ${ }^{22}$

\section{Prioritisation of care}

Prioritisation of illnesses and care for specific diseases should be concordant between patients and their GPs, but may not always be. To date, few studies have addressed prioritisation of care in multimorbid patients. ${ }^{18}{ }^{23}$ We identified a single study that examined the prioritisation of care in multimorbid patients from the patients' perspective and without an index condition. ${ }^{23}$ Health outcome priorities (staying alive, maintaining independence, reducing/eliminating pain and reducing/eliminating symptoms) were evaluated. Maintaining independence was ranked as the most important factor by $76 \%$ of the patients. Among those who ranked maintaining independence as the primary factor, $67 \%$ ranked symptoms relief as the second. Among those who ranked staying alive as their first concern (11\%), 66\% ranked maintaining independence as their second concern. A single prospective study including 92 providers and 1004 US veterans examined the correlation between GPs' and patient's prioritisation of health conditions. ${ }^{18}$ Patients and GPs provided the three most important concerns among a list of nine conditions chosen based on their prevalence (symptomatic chronic conditions: pain, depression, breathing difficulties; and asymptomatic chronic conditions: hypertension, diabetes, hyperlipidaemia, heart disease or heart failure, obesity and tobacco use). In $72 \%$ of cases, the patient's most important concern was represented in the GP's list of three most important concerns. In $60 \%$ of the cases, there was a concordance between the patient's and the GP's prioritisation. Concordance was lower among patients who reported poor health status. ${ }^{18}$

Our aim is to assess the burden related to multimorbidity and to describe prioritisation in the management of multiple chronic conditions in the context of primary care practice, taking patients' and GPs' perspectives into account.

\section{METHODS AND ANALYSES \\ Study objectives}

Primary objective 1: Burden related to multimorbidity-We will assess the importance of the burden related to multimorbidity (ie, disease-related burden and burden of treatment) among patients in a primary care setting, identify the determinants of an increased burden related to multimorbidity, and examine the agreement between patients' and GPs' perceptions of this burden.

Primary objective 2: Prioritisation of care-We want to evaluate the concordance between the problems related to the prioritisation of treatment for multiple chronic conditions as assessed by GPs and as perceived by patients. We will assess prioritisation established by patients and by GPs in the handling of chronic conditions among multimorbid patients in primary care, and identify patient-related or GP-related determinants that influence this prioritisation achieved by patients and GPs, respectively.

Secondary objective: Gender study—We will examine whether the gender of GPs or patients is a determinant of variations in patients' and GPs' perceived burden in relation to multimorbidity, and its related treatment in primary care.

\section{Study design}

This is an observational study with a cross-sectional design.

\section{Study population}

Participants: The study will involve GPs throughout Switzerland and their multimorbid patients. We will enrol 1000 patients through a convenience sample of 100 GPs in private practice. Each participating Institute of Family Medicine will organise the recruitment of 20 GPs from small or large practices in both urban and rural settings, in their respective regions of Switzerland (figure 1). As no Institute of Family Medicine exists in the Italian-speaking part of the country, we restricted our study to the French-speaking and German-speaking regions.

Eligibility: All multimorbid patients visiting a participating GP during the inclusion period and meeting the inclusion criteria are eligible. We considered three chronic conditions as the minimum number of conditions for which a prioritisation of care could be necessary. Inclusion criteria

- To be aged 18 years or older;

- To have been followed by the GP for at least 6 months before the index encounter; 


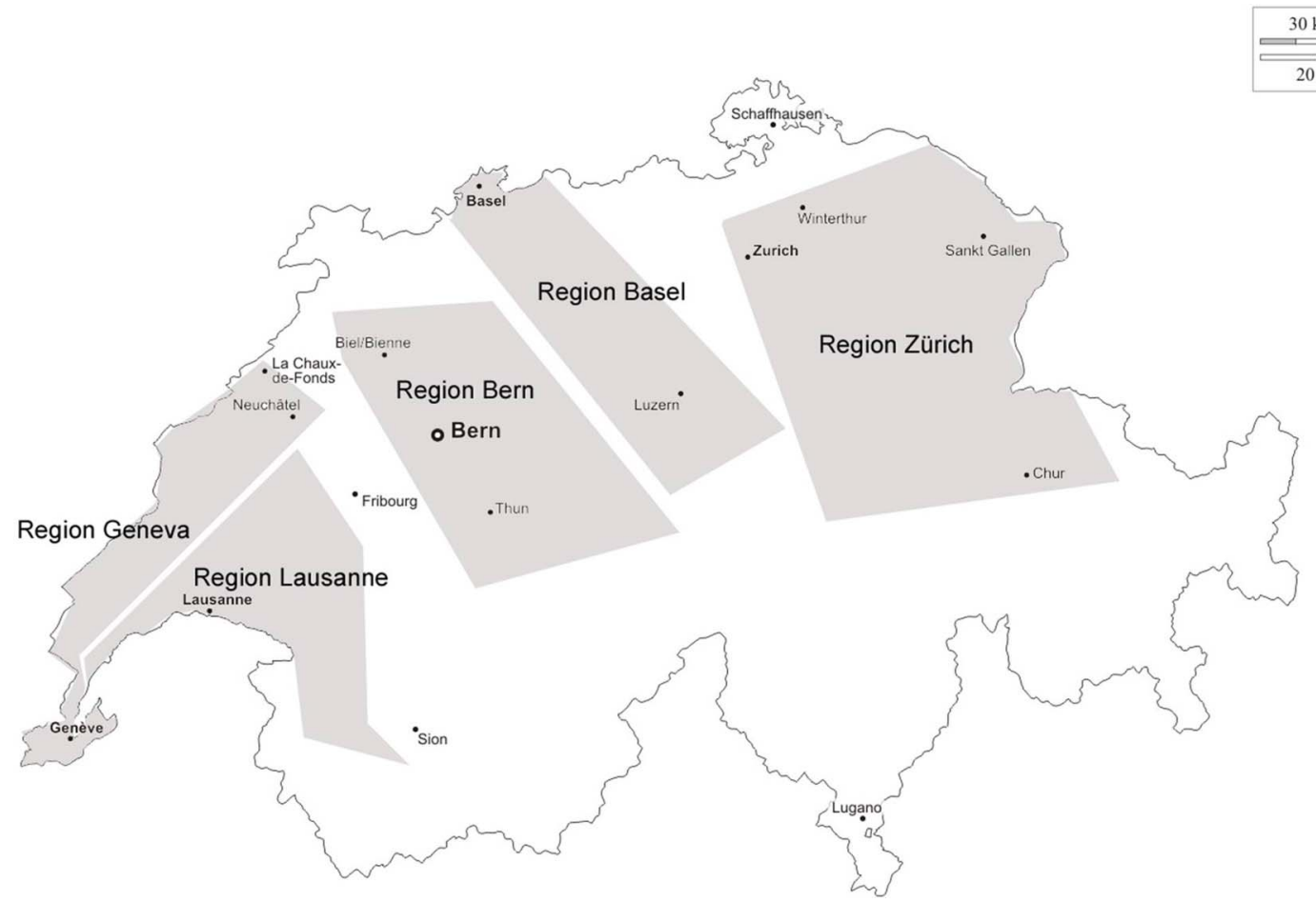

Figure 1 Schematic representation of the regions of Switzerland covered by each Institute of Family Medicine. Each Institute of Family Medicine (Geneva, Lausanne, Bern, Basel, Zürich) will organise the enrolment of 20 general practitioners (GPs) located within its region.

- To have enough knowledge of French or German to be able to complete the questionnaire;

- To suffer from at least three chronic conditions identified from a list of 75 conditions (table 1);

- To consent to participate in the study and to sign the informed consent form.

Table 1 Number of items in each category of the ICPC-2 used to identify multimorbid patients

\begin{tabular}{lc}
\hline ICPC-2 category & $\begin{array}{c}\text { Number of } \\
\text { items }\end{array}$ \\
\hline General and unspecified & 3 \\
Blood, blood forming organs and immune & 3 \\
mechanism & \\
Digestive & 7 \\
Endocrine/metabolic and nutritional & 5 \\
Neurological & 10 \\
Skin & 1 \\
Musculoskeletal & 4 \\
Urological & 3 \\
Respiratory & 4 \\
Eye & 3 \\
Ear & 2 \\
Cardiovascular & 10 \\
Psychological & 17 \\
Female genital & 2 \\
Male genital & 1 \\
\hline ICPC-2, International Classification of Primary Care, V.2.
\end{tabular}

Exclusion criteria

- To suffer from a major cognitive or psychiatric disorder, and as such be unable to answer the questionnaire;

- To be unable to read and/or to understand French or German;

- To suffer from an emergency or acute life-threatening condition (physical or psychological);

- To refuse to participate to the study after initial approval and consent.

\section{Sampling}

Recruitment of patients (figure 2): Each GP will include 10 patients meeting the inclusion criteria. Recruitment will take part over a maximum period of 10 weeks per GP. The data collection process will start on 12 January 2015 and will end on 30 September 2015. Each GP will be provided a randomisation calendar specifying which patient to enrol for each half-day of the recruitment weeks. We selected half-days for the recruitment of patients to match GPs' schedule as accurately as possible, and to take into account the fact that many GPs work either part-time or have a variety of professional activities (teaching, home visits, etc). We wanted to make sure that the GP was seeing patients at his practice during the entire time slot considered. Patients' recruitment is based on patients who are scheduled for each half-day of recruitment. The randomisation calendar gives the ordered number of the consultation to consider, and 


\begin{tabular}{|c|c|}
\hline $\begin{array}{l}\text { Study setup, } \\
\text { enrolment and } \\
\text { training of GPs }\end{array}$ & 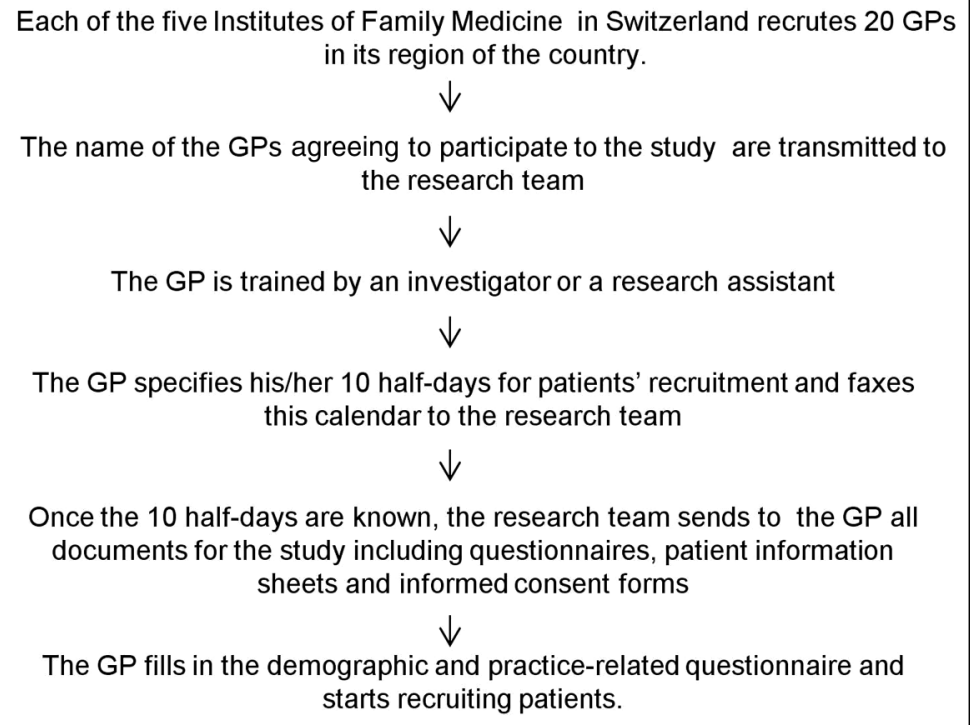 \\
\hline $\begin{array}{c}\text { Patients inclusion } \\
\text { and data collection } \\
\text { process }\end{array}$ & $\begin{array}{l}\text { On each pre-specified half-day of inclusion, the GP identifies the patient to } \\
\text { include using the randomisation calendar } \\
\text { The patient meets the inclusion } \\
\text { criteria }\end{array}$ \\
\hline
\end{tabular}

Figure 2 General practitioners' (GPs') and patients' recruitment process and general study procedure.

the GP includes the patient of this consultation if he/ she meets the inclusion criteria. If the randomised patient does not meet the inclusion criteria, the next patient who fulfils the inclusion criteria is eligible. If no patient is included at the end of the half-day, the recruitment process will be postponed to the next half-day of consultations. The patient will be asked to consent to participate to a telephone interview questionnaire that will take place within $48 \mathrm{~h}$ after the visit to the GP. The patient's name and phone number will then be transmitted to a research assistant. If an eligible patient refuses to participate, their date of birth, sex and reason for refusing participation will be documented.

Procedure (figure 2): Once the GP agrees to participate, he/she will be trained by a research assistant or an investigator to ensure the aim of the study and data collection processes are clearly understood. Training will include detailed information on the procedure to follow, include patients using the randomisation calendar, and the list of 75 conditions provided. GPs will also be instructed on the data collection process using the questionnaires. Data collection from GPs will take place at the GPs' 
Table 2 All variables collected using the three different questionnaires

\begin{tabular}{|c|c|}
\hline Variable & Measure \\
\hline \multicolumn{2}{|c|}{ GP-related variables (paper-based questionnaire) } \\
\hline Age & Date of birth \\
\hline Sex & Sex \\
\hline Place of graduate training & City, country \\
\hline Place of postgraduate training & City, country \\
\hline Type of practice & Independent/on-a-contract GP, group practice, healthcare network \\
\hline Years of practice 1 & Year medical diploma was obtained \\
\hline Years of practice 2 & Year started independent practice \\
\hline Other activity & $\begin{array}{l}\text { Aside from medical practice (eg, research; teaching; practice in a specific context, such } \\
\text { as prison, hospice for elders, school in a company, etc) }\end{array}$ \\
\hline City/countryside & Location of the practice (urban, sub-urban, rural) + zipcode \\
\hline Number of consultations & Mean number of consults per week \\
\hline Mean age of patients & $\begin{array}{l}\text { Mean age of patients in the practice population as determined using the electronic } \\
\text { filing or electronic billing system }\end{array}$ \\
\hline Subspecialty & Medical board specialty other than that of General Internal Medicine \\
\hline \multicolumn{2}{|c|}{ Patient-related variables assessed through the GP survey (paper-based questionnaire) } \\
\hline Age & Date of birth \\
\hline Sex & Sex \\
\hline Visits to GP in the last year & Number of visits to the GP in the last 12 months \\
\hline Visits to GP in the last month & Number of visits to the GP in the last month \\
\hline Time followed by GP & Number of years that the patient has been treated by the GP \\
\hline Hospitalisations & Number of hospitalisations in the last 12 months \\
\hline Number of chronic conditions & Number of chronic conditions from the list provided \\
\hline List of chronic conditions & List of chronic conditions by decreasing order of importance \\
\hline Conditions important to the patient & List of the 3 chronic conditions the most important to the patient, according to the GP \\
\hline Severity of each chronic condition & Evaluated using the cumulative illness rating scale \\
\hline Treatment & Current treatment plan \\
\hline Renouncement & Conditions voluntarily left untreated or suboptimally treated by the GP \\
\hline Interdisciplinarity & Specialist MD/nurse/physiotherapist/rehabilitation therapist/caregiver \\
\hline Treatment burden & $\begin{array}{l}\text { Subjective estimation of the patient's treatment burden on a } 0 \text { to } 10 \text { scale }(0=\text { burden } \\
\text { not important at all; } 10=\text { very important burden) }\end{array}$ \\
\hline \multicolumn{2}{|c|}{ Patient-related variables assessed through the patient survey (telephone interview questionnaire) } \\
\hline Age & Date of birth \\
\hline Sex & Sex \\
\hline Marital status & Patient's marital status (single, married, separated or divorced, widow(er)) \\
\hline Place of education & City, country \\
\hline Mother tongue & Patient's first language(s) \\
\hline Education & $\begin{array}{l}\text { Highest schooling level achieved (primary school, secondary school, practical training, } \\
\text { high school or equivalent, superior degree non-university, university, other) }\end{array}$ \\
\hline Size of the household/living situation & Number of adults ( $\geq 18$ years old) and children ( $<18$ years old) living with the patient \\
\hline Number of MDs & Number of medical doctors (GPs and specialists) involved in the patient's treatment \\
\hline Medical and social assistance & Does the patient receive medical and/or social assistance? \\
\hline Pillbox & Use of a pillbox $(\mathrm{Y} / \mathrm{N})$ \\
\hline Interdisciplinarity & Cleaning help or meal delivery system \\
\hline Health literacy & Health literacy will be assessed using the short 6-item HLS-EU questionnaire \\
\hline Prioritisation & Most important and second most important conditions \\
\hline Quality of life & Quality of life will be assessed using the EQ-5D-3L questionnaire \\
\hline SES & SES will be assessed using the DipCare questionnaire \\
\hline Treatment burden & Treatment burden will be assessed using the treatment burden questionnaire \\
\hline
\end{tabular}

private practices using paper-based questionnaires. For patients, we will use delayed phone interview questionnaires. Each GP will first complete a survey providing demographic and practice-related data. Patient enrolment will take place during a visit to the GP. Randomly identified patients will be informed of the study by their
GP and if they agree to take part, they will provide written informed consent. Enrolled patients will complete a 25 min telephone-based questionnaire. For each patient who is enrolled, the GP will complete a $20 \mathrm{~min}$ paper-based questionnaire. All data will be coded during the data collection process. Patients' names and phone 
numbers will be destroyed after data collection is completed. Research assistants will conduct all patients' telephone interviews, in French or German, from the Institute for Family Medicine in Lausanne. The research team in Lausanne will train the research assistants to conduct the telephone interviews and to collect the data, in collaboration with the Pedagogical Unit of the Faculty of Biology and Medicine of Lausanne. Training will include conducting and handling phone interviews, including the management of negative emotions that may arise during the interview. A patient-actor will participate in this training. The training will include learning to ask questions exactly as written on the questionnaires to ensure homogeneity of the data collection between patients and between research assistants. Research assistants will also be trained to record data using the Epidata V.3.1 software.

\section{Variables}

We will collect GP-related and patient-related variables (table 2). We describe the more complex variables below. All questionnaires for which a German or French version did not exist were translated using a translation/ back-translation procedure.

Patient-related variables assessed through the GP survey include the number and list of chronic conditions, severity of each chronic condition, current treatment and renouncement of treatment.

Number and list of chronic conditions: The International Classification of Primary Care, V.2 (ICPC-2), is a classification of symptoms and diagnostics specifically designed for use in primary care. ${ }^{24}$ We will employ a list of 75 chronic conditions identified in the ICPC-2 following a methodology described elsewhere (N'Goran et al, in preparation). Briefly, we conducted a nationwide survey of GPs using a modified Delphi method. ${ }^{25}$ We used experts from five different regions: Basel, Bern, Geneva, Vaud and Zürich. We started with all 686 items of the ICPC-2 and proceeded to a step-by-step elimination. First, a focus group of five experts removed from the ICPC-2 items that were irrelevant in the context of chronic conditions. Then, three rounds of online survey were conducted: experts had to score the chronic aspect of each remaining item first as chronic, not chronic or in between, and second on a scale of 1-9 (2 rounds); and then assess the relevance of the chronic items in the context of multimorbidity ( 1 round). To analyse data, we used the median values and the inter-percentile range adjusted for symmetry (IPRAS) for agreement/ disagreement between experts. ${ }^{25}$ Items reaching a median value of 6 and agreement between experts were retained in the final list.

Severity of each chronic condition: For each patient enrolled, the GP will provide a quantitative estimate of impairment for each organ area using the cumulative illness rating scale (CIRS). ${ }^{26}$ The CIRS involves rating the impairment of 14 organs or systems. Medical problems are compiled and quantified by organ system, resulting in the computation of a cumulative score that represents the degree of impairment to the whole person. The CIRS has been used in studies involving multimorbid patients and been validated for use in primary care. ${ }^{27-30}$

Current treatment: The GP will provide a list of all medications, preventive measures and rehabilitative measures prescribed. The actual treatment will be considered.

Renouncement of treatment: The GP will provide a list of conditions for which he/she consider the treatment as suboptimal, because it had to be adapted due to potential drug interaction, treatment burden or lack of compliance from the patient or following a shared decision with the patient.

Patient-related variables assessed through the patients' survey include prioritisation, quality of life, SES, health literacy and treatment burden.

Prioritisation: We will assess the prioritisation of chronic conditions from the patient's perspective by asking if he/she had the power to remove one health condition, which one he/she would choose first and then second. It is possible that one or both answers to this question will not refer to chronic conditions; however, we expect this to be the case only for a restricted number of patients.

Quality of life: We will evaluate the burden related to multimorbidity from the patient's perspective using the EQ-5D-3L quality-of-life scale. ${ }^{31-33}$ The EQ-5D-3L is a short, validated questionnaire for the self-assessment of quality of life for which population reference values are available for Switzerland. ${ }^{34} 35$

SES: The SES of enrolled patients will be assessed using the deprivation in primary care questionnaire (DipCare), which was developed and validated in the French-speaking part of Switzerland ${ }^{36}$ and translated into German. An overall score of deprivation will be then computed.

Health literacy: We will use the six-item questionnaire from the European Health Literacy Survey project (HLS-EU 6) to assess health literacy. ${ }^{37}$ These items were selected based on content and difficulty experienced from the 47-question survey (HLS-EU 47). ${ }^{37} 38$ The HLS-EU 6 was developed on a random subsample of the HLS-EU 47 survey $(\mathrm{N}=3411)$, and cross-validated with another random subsample ( $\mathrm{N}=3383)$ (HLS-EU consortium, personal communication, 2014). Answers to each question from the HLS-EU 6 are given in the form of a four-point Likert scale (1=very difficult, $2=$ =fairly difficult, $3=$ fairly easy, $4=$ very easy). Although no population-based data exist for Switzerland, data do exist for neighbouring countries.

Treatment burden: The treatment burden questionnaire (TBQ) was developed recently to assess the overall burden related to the treatment of chronic conditions. ${ }^{39}$ It consists of 13 questions whose answers are each scored from 0 to 10 , with 0 corresponding to no burden and 10 to a very important/considerable burden. The global (summed) score of treatment burden ranges from 0 to 
130. No Swiss population-based data exist for this questionnaire.

\section{Planned analyses}

Primary objective 1: Burden related to multimorbidity

- To identify patient-related or GP-related determinants that influence the disease-related burden (number of chronic conditions, CIRS and EQ-5D-3L) or TBQ

- To analyse the relationship between the perceptions of patients and the GPs regarding the burden related to multimorbidity using the number of chronic conditions identified, the CIRS and the EQ-5D-3L questionnaire

- To assess the burden of treatment in terms of the number of pills prescribed per day, and to test whether it relates to the patients' evaluation using the TBQ and to GPs' evaluation of the burden of treatment

- To assess whether the number of conditions treated is related to TBQ scores using univariate and multivariate models

Primary objective 2: Prioritisation of care

- To evaluate whether in multimorbid patients some chronic conditions are left untreated by comparing the list of chronic conditions diagnosed with the list of treatments prescribed.

- To describe the subgroup of patients with conditions left voluntarily untreated or suboptimally treated, and compare it to the group of patients in which no conditions were left untreated

- To test whether the two conditions classified as most important by GPs and patients are concordant. We will obtain a series of concordant and discordant patients'/GPs' pairs; describe and compare the subgroups of patients/GPs with concordant pairs versus discordant pairs; and evaluate whether the concordance between patients' and GPs' evaluations of the most important chronic conditions correlates with the TBQ scores.
- To identify the patient-related or GP-related determinants that have an influence on concordance in the prioritisation achieved by patients and GPs, respectively.

Secondary objective: Gender study

- To examine whether gender of patients or GPs influences the TBQ or the GPs' evaluation of the burden of treatment

- To examine whether gender influences the concordance between GPs' and patients' prioritisations of chronic conditions

\section{Statistics}

Patients' sample size: The sample size estimation is based on reported proportions of patients with different numbers of chronic conditions from studies conducted in primary care. We evaluated the number of patients whom we would include with different numbers of chronic conditions, given possible sample sizes of 500 , 750 and 1000 patients, if the repartition of patients was similar to that in any of the three studies considered. Based on this data, a sample size of 1000 is enough to allow for potential subgroup analyses. The estimates are reported in table 3 . This sample size is also coherent considering that a statistical model requires approximately 15 patients per variable.

Descriptive statistics: Descriptive statistics will be performed to describe the sample of GPs and the sample of patients. All collected measures and derived variables will be summarised using usual descriptive statistics and the associated 95\% CI. For categorical variables, the description will consist (at minimum) of the sample size; numbers of missing data; valid data, median, upper and lower quartiles; and the frequency and percentage of each category.

Exploratory data analysis: t-Test will be used for continuous normally distributed variables; one-way analysis of variance will be used if we need to compare more than two groups. For continuous variables not normally distributed,

Table 3 Estimates for sample size evaluation

\begin{tabular}{|c|c|c|c|c|c|c|c|c|}
\hline \multirow[b]{2}{*}{ Reference } & \multirow[b]{2}{*}{$\begin{array}{l}\text { Coding } \\
\text { system } \\
\text { used }\end{array}$} & \multirow[b]{2}{*}{ Age, years } & \multirow[b]{2}{*}{$\begin{array}{l}\text { Number of } \\
\text { chronic } \\
\text { conditions }\end{array}$} & \multirow[b]{2}{*}{$\begin{array}{l}\text { Number of } \\
\text { patients }\end{array}$} & \multirow[b]{2}{*}{ Percentage } & \multicolumn{3}{|c|}{ Expected number } \\
\hline & & & & & & $\begin{array}{l}\text { Sample } \\
\text { size of } 500\end{array}$ & $\begin{array}{l}\text { Sample } \\
\text { size of } 750\end{array}$ & $\begin{array}{l}\text { Sample } \\
\text { size of } \\
1000\end{array}$ \\
\hline \multirow[t]{6}{*}{ Barnett et al, $2012^{2}$} & Read codes & No age limit & 3 & 99487 & 0.418 & 209 & 314 & 418 \\
\hline & & & 4 & 60417 & 0.254 & 127 & 190 & 254 \\
\hline & & & 5 & 35641 & 0.150 & 75 & 112 & 150 \\
\hline & & & 6 & 20507 & 0.086 & 43 & 65 & 86 \\
\hline & & & 7 & 11080 & 0.047 & 23 & 35 & 47 \\
\hline & & & $\geq 8$ & 10846 & 0.046 & 23 & 34 & 46 \\
\hline \multirow[t]{2}{*}{ Rizza et al, $2012^{40}$} & ICPC-2 & $\geq 20$ & 3 & 2319 & 0.48 & 240 & 360 & 480 \\
\hline & & & $>3$ & 2513 & 0.52 & 260 & 187 & 520 \\
\hline \multirow[t]{3}{*}{ Glynn et al, $2011^{5}$} & ICPC-2 & $>50$ & 3 & 636 & 0.47 & 233 & 349 & 466 \\
\hline & & & 4 & 367 & 0.27 & 134 & 202 & 269 \\
\hline & & & $\geq 5$ & 362 & 0.27 & 133 & 199 & 265 \\
\hline
\end{tabular}


we will use non-parametric tests: the Mann-Whitney U test to compare two groups, and the Kruskall-Wallis test to compare three or more groups. For categorical variables, we will employ the $\chi^{2}$ test or Fisher's exact test, depending on the number of observations obtained in each category considered. All tests are two-sided unless otherwise specified, and $\mathrm{p}<0.05$ will be considered statistically significant. Correlation between variables will be assessed using the Pearson correlation coefficient for normally distributed variables or the Spearman correlation otherwise. We will evaluate the level of agreement between two of the same parameter using agreement tables, scatter plots and Cohen's $\kappa$ statistic.

Univariate tests will be used to select variables according to the $p$ value obtained $(p<0.25)$ to include in the multivariate analyses. For the multivariate analyses, generalised linear regression models will be used: Poisson regression for outcome variables in the form of scores, multinomial logistic regression for non-dichotomous categorical variables, or logistic regression for dichotomous variables, as appropriate. Mixed effects multilevel models that take clustering and between-site differences into account will be considered. These models will include two levels of clustering in order to take into account the variability caused by regions and GPs. Models will be compared and the best model will be selected on the basis of likelihood ratio tests.

\section{Ethical aspects}

The study protocol and study documentation, including all questionnaires and the informed consent, were accepted by the ethical committee of the Canton of Vaud acting as the lead ethical committee for Switzerland. The protocol was then transmitted to the local ethical commissions of the different regions involved in the study.

We anticipate no direct benefits for the patients. Indirect benefits include contribution to understanding the mechanisms underlying the handling of patients with multimorbidity, which will eventually improve the management of patients. No important risk is foreseen as a direct result of the survey. GPs will handle the healthcare of included patients as normal.

\section{Potential biases and limits}

We will use a convenience sample of 100 GPs, and thus will not follow a randomised sampling for the identification of GPs. As a result, participating GPs may not be representative of the overall population of GPs practising in Switzerland. Sociodemographic data collected, such as sex, age and location of practice, will allow for the characterisation of the participating GPs and the evaluation of external validity. Recruitment of patients will follow a strict randomised procedure, and no selection bias is foreseen. In addition, demographic data will be collected from patients who refuse to participate in the study, as well as their reasons for non-participation. Data will mainly be collected through self-assessment questionnaires. This leaves the potential for information bias. However, as both GPs and patients will have no detailed knowledge of the objectives of the study, or of the analyses planned, we anticipate that information bias will not be significant or will only lead to non-differential misclassification. We will use standardised tests of proven high internal validity. However, for some tests, such as the CIRS, the TBQ and the HLS-EU 6 questions, no data in Switzerland have been reported, as yet. For tests for which no Swiss data are available, data from populations close to the Swiss population (German and French speaking) are available. The TBQ test was developed and used in a single French population. No German version has been developed and used previously.

\section{Pilot phase}

A pilot phase is planned for this study. We will enrol five pilot patients through a convenience sample of five GPs across Switzerland based on their willingness to participate. We will ask each patient to complete the telephone interview questionnaire and each GP to complete the paper questionnaire. The pilot phase aims to evaluate the feasibility of the study, to recognise and solve potential issues with the use of questionnaires, and to better define specific aspects of the training of research assistants.

Author affiliations

${ }^{1}$ Institute of Family Medicine, University of Lausanne, Lausanne, Vaud, Switzerland

${ }^{2}$ Institute of Primary Care, University of Zürich, Zürich, Switzerland

${ }^{3}$ Institute of Primary Care at the University of Berne (BIHAM), University of Berne, Berne, Switzerland

${ }^{4}$ Centre for Primary Health Care, University of Basel, Basel, Switzerland

${ }^{5}$ Primary Care Unit, Faculty of Medicine, University of Geneva, Geneva, Switzerland

${ }^{6}$ Institute of Social and Preventive Medicine, Lausanne University Hospital, Lausanne, Vaud, Switzerland

${ }^{7}$ Department of Ambulatory Care and Community Medicine, Lausanne University Hospital, Lausanne, Vaud, Switzerland

Contributors All the authors developed the study's objectives and defined the list of variables to be collected. AD-L, AAN, BB, PB, NS, DW, LH, PF and RT developed the questionnaires in French and German. AD-L, AAN and $L H$ drafted the protocol and oversaw submission of the protocol to the ethics commission. AD-L, AAN and LH developed the necessary study documents for the ethics commission. All authors contributed to the revision of the manuscript and the decision to submit for publication.

Funding The Swiss University Conference granted funding to reinforce teaching and research in primary care in Switzerland. Within the framework of this financial support, the five Swiss institutes for Family Medicine (Basel, Bern, Geneva, Zürich and Lausanne) collaborated under the denomination: Swiss Academy for Family Medicine (SAFMED). Part of the funding is to be used for national research projects in primary care.

Competing interests None declared.

Patient consent Obtained.

Ethics approval Research ethics committee of Canton Vaud, Switzerland (Protocol 315/14).

Provenance and peer review Not commissioned; externally peer reviewed.

Open Access This is an Open Access article distributed in accordance with the Creative Commons Attribution Non Commercial (CC BY-NC 4.0) license, 
which permits others to distribute, remix, adapt, build upon this work noncommercially, and license their derivative works on different terms, provided the original work is properly cited and the use is non-commercial. See: http:// creativecommons.org/licenses/by-nc/4.0/

\section{REFERENCES}

1. Van den Akker M, Buntinx F, Knottnerus JA. Comorbidity or multimorbidity: what's in a name? A review of literature. Eur J Gen Pract 1996;2:65-70.

2. Barnett K, Mercer SW, Norbury M, et al. Epidemiology of multimorbidity and implications for health care, research, and medical education: a cross-sectional study. Lancet 2012;380:37-43.

3. van Oostrom SH, Picavet HS, van Gelder BM, et al. Multimorbidity and comorbidity in the Dutch population-data from general practices. BMC Public Health 2012;12:715.

4. Garcia-Olmos L, Salvador $\mathrm{CH}$, Alberquilla A, et al. Comorbidity patterns in patients with chronic diseases in general practice. PLoS ONE 2012;7:e32141.

5. Glynn LG, Valderas JM, Healy P, et al. The prevalence of multimorbidity in primary care and its effect on health care utilization and cost. Fam Pract 2011;28:516-23.

6. Marengoni A, Winblad B, Karp A, et al. Prevalence of chronic diseases and multimorbidity among the elderly population in Sweden. Am J Public Health 2008;98:1198-200.

7. Brett T, Arnold-Reed DE, Popescu A, et al. Multimorbidity in patients attending 2 Australian primary care practices. Ann Fam Med 2013;11:535-42.

8. Vogeli C, Shields AE, Lee TA, et al. Multiple chronic conditions: prevalence, health consequences, and implications for quality, care management, and costs. J Gen Intern Med 2007;22(Suppl 3):391-5.

9. Fortin M, Stewart M, Poitras ME, et al. A systematic review of prevalence studies on multimorbidity: toward a more uniform methodology. Ann Fam Med 2012;10:142-51.

10. Schafer I, Hansen H, Schon G, et al. The influence of age, gender and socio-economic status on multimorbidity patterns in primary care. First results from the multicare cohort study. BMC Health Serv Res 2012;12:89.

11. Guthrie B, Payne K, Alderson $\mathrm{P}$, et al. Adapting clinical guidelines to take account of multimorbidity. BMJ 2012;345:e6341.

12. Uijen AA, van de Lisdonk EH. Multimorbidity in primary care: prevalence and trend over the last 20 years. Eur $J$ Gen Pract 2008;14(Suppl 1):28-32.

13. Gijsen R, Hoeymans N, Schellevis FG, et al. Causes and consequences of comorbidity: a review. J Clin Epidemiol 2001:54:661-74.

14. Bayliss EA, Bayliss MS, Ware JE Jr, et al. Predicting declines in physical function in persons with multiple chronic medical conditions: what we can learn from the medical problem list. Health Qual Life Outcomes 2004;2:47.

15. University SECatB. Patterns of medication use in the United States, Report from the Slone Survey. Secondary Patterns of medication use in the United States, Report from the Slone Survey. 2006.

16. Holmes HM, Min LC, Yee M, et al. Rationalizing prescribing for older patients with multimorbidity: considering time to benefit. Drugs Aging 2013;30:655-66.

17. Koberlein J, Gottschall M, Czarnecki K, et al. General practitioners' views on polypharmacy and its consequences for patient health care. BMC Fam Pract 2013;14:119.

18. Zulman DM, Kerr EA, Hofer TP, et al. Patient-provider concordance in the prioritization of health conditions among hypertensive diabetes patients. J Gen Intern Med 2010;25:408-14.

19. Fortin M, Lapointe L, Hudon C, et al. Multimorbidity is common to family practice: is it commonly researched? Can Fam Physician 2005;51:244-5.
20. Ostbye T, Yarnall KS, Krause KM, et al. Is there time for management of patients with chronic diseases in primary care? Ann Fam Med 2005;3:209-14.

21. Boyd CM, Darer J, Boult C, et al. Clinical practice guidelines and quality of care for older patients with multiple comorbid diseases: implications for pay for performance. JAMA 2005;294: 716-24.

22. Sinnott C, Mc Hugh S, Browne J, et al. GPs' perspectives on the management of patients with multimorbidity: systematic review and synthesis of qualitative research. BMJ Open 2013;3: e003610.

23. Fried TR, Tinetti ME, lannone $\mathrm{L}$, et al. Health outcome prioritization as a tool for decision making among older persons with multiple chronic conditions. Arch Intern Med 2011;171:1854-6.

24. Bentsen BG. International classification of primary care. Scand $J$ Prim Health Care 1986;4:43-50.

25. Fitch K, Bernstein SJ, Aguilar MD, et al. The RAND/UCLA Appropriateness Method-User's Manual. In: Corporation R, ed. 2001. http://www.rand.org/content/dam/rand/pubs/monograph_ reports/2011/MR1269.pdf

26. Linn BS, Linn MW, Gurel L. Cumulative illness rating scale. J Am Geriatr Soc 1968;16:622-6.

27. Huntley AL, Johnson R, Purdy $\mathrm{S}$, et al. Measures of multimorbidity and morbidity burden for use in primary care and community settings: a systematic review and guide. Ann Fam Med 2012;10:134-41.

28. Fortin M, Dubois MF, Hudon C, et al. Multimorbidity and quality of life: a closer look. Health Qual Life Outcomes 2007;5:52.

29. Hudon C, Fortin M, Vanasse A. Cumulative Illness Rating Scale was a reliable and valid index in a family practice context. $J$ Clin Epidemiol 2005;58:603-8.

30. Fortin M, Hudon C, Haggerty J, et al. Prevalence estimates of multimorbidity: a comparative study of two sources. BMC Health Serv Res 2010;10:111.

31. EuroQol G. EuroQol-a new facility for the measurement of health-related quality of life. Health Policy 1990;16:199-208.

32. Brooks R. EuroQol: the current state of play. Health Policy 1996;37:53-72.

33. Rabin R, de Charro F. EQ-5D: a measure of health status from the EuroQol Group. Ann Med 2001;33:337-43.

34. Perneger TV, Combescure C, Courvoisier DS. General population reference values for the French version of the EuroQol EQ-5D health utility instrument. Value Health 2010;13. 631-5.

35. Matter-Walstra K, Klingbiel D, Szucs T, et al. Using the EuroQol EQ-5D in Swiss cancer patients, which value set should be applied? Pharmacoeconomics 2014;32:591-9.

36. Vaucher $\mathrm{P}$, Bischoff $\mathrm{T}$, Diserens $\mathrm{EA}$, et al. Detecting and measuring deprivation in primary care: development, reliability and validity of a self-reported questionnaire: the DiPCare-Q. BMJ Open 2012;2: e000692.

37. HLS-EU Consortium (2012). Comparative report of health literacy in eight EU member states. The European Health Literacy Survey HLS-EU (first revised and extended version 5 July 2013). http:// www.health-literacy.eu (accessed May 2015).

38. Sorensen K, Van den Broucke S, Pelikan JM, et al. Measuring health literacy in populations: illuminating the design and development process of the European Health Literacy Survey Questionnaire (HLS-EU-Q). BMC Public Health 2013;13:948.

39. Tran VT, Montori VM, Eton DT, et al. Development and description of measurement properties of an instrument to assess treatment burden among patients with multiple chronic conditions. BMC Med 2012;10:68.

40. Rizza A, Kaplan V, Senn O, et al. Age- and gender-related prevalence of multimorbidity in primary care: the Swiss FIRE project. BMC Fam Pract 2012;13:113. 